\title{
Steps Towards Energy Efficiency in Elastically Actuated Robots
}

\author{
Gianluca Garofalo* and Christian Ott \\ Institute of Robotics and Mechatronics, German Aerospace Center (DLR), \\ Wessling, 82234, Germany \\ * E-mail: gianluca.garofalo(at)dlr.de \\ www.dlr.de/rmc/rm
}

Soft robotic systems - Locomotion, posture and motion control

\begin{abstract}
Elastically actuated robots can store energy in the system and therefore have the potential of efficiently performing tasks. The goal here is to show how the control approach that we have developed, can provide a clear way to use such an energy. The natural capability of this class of system of producing oscillation makes them especially appealing when periodic motions are needed to be generated. Robotic locomotion is an example of application.
\end{abstract}

Keywords: Limit cycle control; Elastically actuated robots; Energy functions.

\section{Motivation}

Elastically actuated robots are increasing in popularity, since the energy stored in the elastic elements can be exploited to achieve higher velocity and reduce the effort of the motors. However, the mechanical design of such systems introduces often additional nonlinearities in the system dynamics which, along with the underactuation problem, make the controller design more challenging. This is probably the reason why a clear methodology that can take advantage of the springs present in the systems is yet not well defined. Here we suggest a possible approach towards energy efficiency when using such systems. To this end we consider our developed controller for regulation of the energy of the system, in which the potential energy of the elastic elements present in the joint is directly taken into account. Clearly, elastically actuated robots inherently offer the possibility to produce oscillation and, when succeeding in utilizing the energy that they store, produce oscillations efficiently. 


\section{Control Approach}

Elastically actuated robots are underactuated system that, assuming to have negligible coupling inertias between motor and link side (a typical assumption which is fulfilled in presence of high gear ratios), can be modeled as

$$
\begin{aligned}
& \mathbf{M}(\mathbf{q}) \ddot{\mathbf{q}}+\mathbf{C}(\mathbf{q}, \dot{\mathbf{q}}) \dot{\mathbf{q}}+\mathbf{g}(\mathbf{q})=\boldsymbol{\tau}(\boldsymbol{\theta}-\mathbf{q}) \\
& \mathbf{B} \boldsymbol{\theta}+\boldsymbol{\tau}(\boldsymbol{\theta}-\mathbf{q})=\boldsymbol{\tau}_{m} .
\end{aligned}
$$

Using a fast torque control loop for the motor dynamics, the torque produced by the springs can be assumed to be the control input for Eq. (1a). The goal is then to produce a limit cycle using the energy of the springs. For a 1 - DoF system regulating the energy to a constant positive value results in an asymptotically stable limit cycle. The basic idea of the controller we proposed in our previous work ${ }^{1}$ is then to produce a limit cycle for a $n$-DoF system by first introducing $n-1$ virtual constraints and then regulating the energy to a desired value, as shown in Fig. 1.

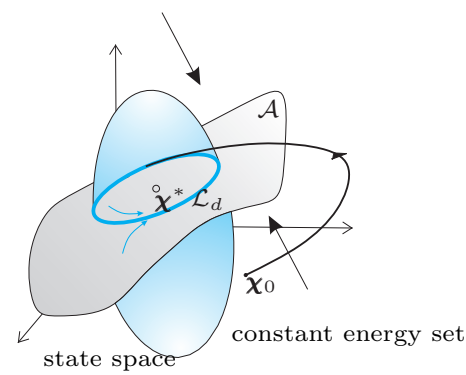

Fig. 1. Conceptual illustration of the idea behind our energy based controller. ${ }^{1}$ The limit cycle is generated regulating an energy function, after that the system has been forced to evolve on a submanifold of the configuration space.

\section{Choice of the Potential Function}

The energy based controller ${ }^{1}$ regulates an energy function given by the kinetic energy of the links and a virtual potential. In order to exploit the presence of the elastic elements, we propose a potential function which takes into account the physical energy of the system:

$$
U(\mathbf{q})=U_{g}(\mathbf{q})+U_{k}\left(\boldsymbol{\theta}_{d}-\mathbf{q}\right)-U_{g}\left(\mathbf{q}_{d}\right)-U_{k}\left(\boldsymbol{\theta}_{d}-\mathbf{q}_{d}\right)
$$


where $U_{g}(\mathbf{q})$ is the gravitational potential energy, $U_{k}(\boldsymbol{\theta}-\mathbf{q})$ is the elastic potential present in the system. In order to guarantee the existence of an asymptotically stable limit cycle, the constant motor position $\boldsymbol{\theta}_{d}$ and the constant link position $\mathbf{q}_{d}$ have to be chosen in such a way that the conditions:

- $\mathbf{x}\left(\mathbf{q}_{d}\right)=\mathbf{0}$

- $U(\mathbf{q}) \geq 0, \forall \mathbf{q}: \mathbf{x}(\mathbf{q})=\mathbf{0}$

- $U(\mathbf{q})=0 \Longleftrightarrow \mathbf{q}=\mathbf{q}_{d}, \forall \mathbf{q}: \mathbf{x}(\mathbf{q})=\mathbf{0}$.

are satisfied, ${ }^{1}$ where $\mathbf{x}(\mathbf{q})=\mathbf{0}$ defines the submanifold in which we produce the limit cycle. This is possible whenever the following choice is made:

$$
\mathbf{q}_{d}=\arg \min _{\mathbf{q}} U_{g}(\mathbf{q})+U_{k}\left(\boldsymbol{\theta}_{d}-\mathbf{q}\right) \text {. }
$$

\section{Controller Structure}

The final structure of the controller is depicted in Fig. 2, where three contribution can be distinguished: a dynamic reshaping of the system, the torque responsible for forcing the system to evolve on a 1 - dimensional submanifold of the configuration space and finally the one creating the asymptotically stable limit cycle in the nullspace of the virtual constraint. The last two are dynamically decoupled from each other, while the dynamic reshaping part of the controller compensates for the gravity torque and shifts energy from the constraint space to the nullspace through a power conserving term $\mathbf{F}_{\alpha}$.

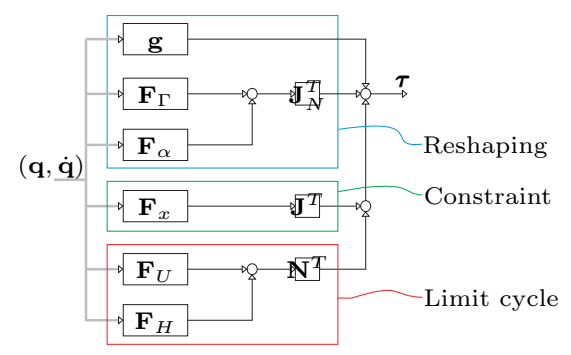

Fig. 2. Controller structure.

\section{References}

1. G. Garofalo, C. Ott and A. Albu-Schäffer, Orbital stabilization of mechanical systems through semidefinite Lyapunov functions, in American Control Conference (ACC), (Washington DC, USA, 2013). 